CARTA AL EDITOR

\section{RÉPLICA: CONSIDERACIONES METODOLÓGICAS SOBRE EL USO DE TERAPIAS DE MEDICINA ALTERNATIVA Y COMPLEMENTARIA EN LA PROVINCIA DE CORONEL PORTILLO, UCAYALI, PERÚ}

REPLY: METHODOLOGICAL CONSIDERATIONS
ON THE USE OF ALTERNATIVE AND COMPLE-
MENTARY MEDICINE THERAPIES IN THE PROV-
INCE OF CORONEL PORTILLO, UCAYALI, PERU

Rocío Santiváñez-Acosta(1,a, Félix Valenzuela-Oré (1) 1,2,b, Yolanda Ángulo-Bazán (101,c

1 Centro Nacional de Salud Intercultural, Instituto Nacional de Salud, Lima, Perú.

${ }^{2}$ Facultad de Ciencias de la Salud, Universidad Peruana Los Andes, Huancayo, Perú.

a Química farmacéutica; ${ }^{\mathrm{b}}$ licenciado en Enfermería, doctor en Salud Pública; ${ }^{\mathrm{c}}$ médica cirujana

Sr. Editor: Hemos recibido con interés la carta denominada consideraciones metodológicas sobre el artículo «Uso de terapias de medicina alternativa y complementaria en la provincia de Coronel Portillo, Ucayali, Perú»; por lo que procedemos a contestar las observaciones enviadas.

Las fórmulas de cálculo de tamaño muestral tienen como objetivo ayudar a los investigadores a obtener un tamaño de muestra suficiente, que permita un nivel adecuado de precisión al momento de calcular inferencias hacia la población de estudio $^{(1)}$. Contrario a lo que los autores señalan en la carta, el uso de un tamaño de muestra mayor al calculado incrementa el poder estadístico del estudio, lo que aumenta la probabilidad de obtener un resultado estadísticamente significativo cuando el efecto realmente existe ${ }^{(2)}$. Del mismo modo, los autores buscan sustentar que el uso de un tamaño muestral mayor al calculado podría aumentar la probabilidad de error tipo 1 en el estudio, lo que consideramos incorrecto; ya que se conoce que la única manera de mejorar simultáneamente el control sobre los errores tipo 1, tipo 2, y detectar diferencias estadísticas pequeñas pero significativas, es incrementando el tamaño muestral ${ }^{(3)}$.

La población de estudio, por las características geográficas y culturales descritas en nuestra publicación, se puede considerar como una población concentrada y asequible para los investigadores, lo que facilitó el proceso de recolección de

Citar como: Santivañez-Acosta RM, Valenzuela-Oré F, Ángulo-Bazán Y. Replica: Consideraciones metodológicas sobre el artículo "Uso de terapias de medicina alternativa y complementaria en la provincia de Coronel Portillo, Ucayali, Perú". Rev Peru Med Exp Salud Publica. 2021;38(1):190. doi: https://doi.org/10.17843/ rpmesp.2021.381.6743.

Correspondencia: Rocío Santiváñez Acosta; roxioxanti@yahoo.com Av. Defensores del Morro 2268, Lima 9, Perú; roxioxanti@yahoo.com

Recibido: 12/11/2020 Aprobado: 18/11/2020 En línea: 03/02/2020 datos y ayudó a obtener el tamaño muestral final. Esta situación no afecta significativamente el plan de análisis del estudio, y ya ha sido mostrada en otra publicación previa ${ }^{(4)}$. La oportunidad de trabajar con un tamaño muestral mayor al calculado es una situación inusual pero valiosa para ofrecer resultados más precisos y de mayor utilidad para futuras investigaciones; siempre y cuando se cumpla estrictamente con otros aspectos metodológicos, como la selección muestral.

Por otro lado, la decisión de asumir una prevalencia esperada del $50 \%$, en casos en los que ésta no sea conocida en la población de estudio, se ampara justamente en asegurar un mayor tamaño muestral para disminuir la probabilidad de error en las estimaciones ${ }^{(5)}$, lo que consideramos como una decisión válida para esta población en particular y en la que no existe antecedentes previos sobre el tema de estudio.

Adicionalmente, entendemos que podría haber un error de interpretación en el párrafo de nuestro manuscrito en el que se habla del cálculo del tamaño muestral; ya que, se consignan los estimadores necesarios para el cálculo del tamaño muestral, lo que es coherente con lo mostrado en la carta. Sin embargo, aclaramos que el tamaño final con el que se trabajó en el estudio fue de 840 , según lo sustentado en párrafos anteriores.

Finalmente, recomendamos que los autores de la carta redoblen cuidados con la selección de las fuentes de información que citan en su argumento teórico, ya que uno de los estudios referenciados (Suresh, 2012) fue retractado por copiar secciones enteras de texto de una publicación previa que tenía otro objetivo (aleatorización en ensayos clínicos) ${ }^{(6)}$.

Contribuciones de autoría: RSA, FVO y YEAB han participado en la concepción de la réplica a la carta al editor, la recolección de información, su redacción y aprobación de la versión final.

Conflictos de interés: Los autores declaran no tener conflictos de interés.

Financiamiento: Autofinanciado

\section{REFERENCIAS BIBLIOGRÁFICAS}

1. Pourhoseingholi MA, Vahedi M, Rahimzadeh M. Sample size calculation in medical studies. Gastroenterol Hepatol Bed Bench. 2013;6(1):14-7.

2. Indrayan A, Malhotra RK. Confidence Intervals, Principles of Tests of Significance, and Sample Size. En: Medical Biostatistics. 4th edition. Boca Raton: Chapman and Hall/CRC; 2017. p. 280-1.

3. White S. Research Questions About One Group. En: Basic \& Clinical Biostatistics: Fifth Edition. New York: McGraw-Hill Education; 2019.

4. Morales Quispe J, Añez Ramos RJ, Suarez Oré CA. Nivel de actividad física en adolescentes de un distrito de la región Callao. Rev Per Med Exp Salud Publica. 2016;33(3):471-7. doi:10.17843/rpmesp.2016.333.2312.

5. Macfarlane SB. Conducting a Descriptive Survey: 2. Choosing a Sampling Strategy. Trop Doct. 1997;27(1):14-21.doi:10.1177/004947559702700108.

6. Patil M. Retraction: Sample size estimation and power analysis for clinical research studies. J Hum Reprod Sci. 2015;8(3):186. 